\title{
Cystic fibrosis testing 8 years on: Lessons learned from carrier screening and sequencing analysis
}

\author{
Charles M. Strom, MD, PhD ${ }^{l}$, Beryl Crossley, $M D^{I}$, Arlene Buller-Buerkle, PhD $D^{I}$, Michael Jarvis, PhD ${ }^{I}$, \\ Franklin Quan, $P h D^{l}$, Mei Peng, $P h D^{I}$, Kasinathan Muralidharan, PhD ${ }^{2}$, Victoria Pratt, PhD ${ }^{2}$, \\ Joy B. Redman, MS, MBA ${ }^{I}$, and Weimin Sun, $P h D^{1}$
}

\begin{abstract}
Purpose: This study reviews data from our cystic fibrosis testing program to evaluate the performance of population-based carrier screening and compare observed detection rates with predicted results of the American College of Medical Genetics/American College of Obstetricians and Gynecologists recommended panel of 23 mutations. Methods: We queried our proprietary databases containing approximately 3 million cystic fibrosis screening tests, 1300 prenatal diagnostic tests, and 2400 cystic fibrosis sequencing analyses. Results: We observed an overall cystic fibrosis carrier frequency of 1:37.6 individuals in the pan-ethnic tested population. This represents a detection rate of $77 \%$, given an estimated US pan-ethnic carrier frequency of 1:29. For patients self-identified as white or Ashkenazi Jewish, a carrier frequency of 1:29 and 1:27 were observed, respectively. A combined frequency of $1: 28$, representing close to $90 \%$ of carriers, was identified in these two highest risk populations. In total, 119 affected fetuses were identified by prenatal diagnoses, a ratio of 1 affected fetus per 25,000 carrier screens. Of 62 newborns with positive immunoreactive trypsinogen and positive sweat tests, almost all of whom had been tested using the American College of Medical Genetics/American College of Obstetricians and Gynecologists panel, only two individuals would have been identified using an expanded mutation panel. Conclusion: The American College of Medical Genetics/American College of Obstetricians and Gynecologists panel of 23 mutations is performing as predicted in detecting cystic fibrosis carriers in the United States among all ethnic groups. No recurrent mutations have been detected in sufficient numbers to justify including any additional mutations to the existing panel. An expanded American College of Medical Genetics/American College of Obstetricians and Gynecologists panel would have a minimal impact on the prevention of births of children affected with cystic fibrosis. Genet Med 2011:13(2):166-172.
\end{abstract}

Key Words: cystic fibrosis, carrier screening, DNA sequencing, population, screening

$\mathrm{C}$ ystic fibrosis $(\mathrm{CF})$ is the most common life-limiting inherited disease among individuals of white ancestry. ${ }^{1-3}$ The CF Foundation estimates that there are 30,000 individuals affected with CF in the United States and that 1:29 Americans are CF carriers. ${ }^{1}$ Patients with classical CF suffer from a constel-

From the ${ }^{1}$ Nichols Institute, Quest Diagnostics, San Juan Capistrano, California; and ${ }^{2}$ Nichols Institute, Quest Diagnostics, Chantilly, Virginia.

Charles M. Strom, MD, PhD, Genetic Testing Center, Nichols Institute Quest Diagnostics, 33608 Ortega Highway, San Juan Capistrano, CA 926752042. E-mail: charles.m.strom@questdiagnostics.com.

Disclosure: All authors are full time employees at Quest Diagnostics, a commercial laboratory that provides testing services.

Submitted for publication June 1, 2010.

Accepted for publication August 31, 2010.

Published online ahead of print January 10, 2011.

DOI: $10.1097 /$ GIM.0b013e3181fa24c4 lation of symptoms including exocrine pancreatic insufficiency, chronic lung disease, and congenital bilateral absence of the vas deferens (CBAVD) in males. There are many forms of milder $\mathrm{CF}$ that range from lung disease without pancreatic disease (pancreatic sufficient CF), isolated pancreatitis, chronic sinus, and/or pulmonary disease in adults, to isolated CBAVD. Postnatal diagnosis of $\mathrm{CF}$ relies on a combination of clinical observation and laboratory testing. ${ }^{3}$

Almost all cases of $\mathrm{CF}$ are presumed to be caused by mutations in the $\mathrm{CF}$ transmembrane regulatory $(C F T R)$ gene that encodes CFTR protein, which helps regulate chloride ion flux. ${ }^{4}$ Almost all patients with classical CF will have diagnostic elevations of chloride ion in their sweat after the newborn period. ${ }^{3}$

Newborn screening for CF has been instituted in many states using immunoreactive trypsinogen (IRT). This is a screening test, and IRT alone does not distinguish between patients with classical CF, patients with milder forms, and CF carriers. ${ }^{5-7}$ A combination of clinical observation, the sweat chloride test, and molecular diagnostics are required for a postnatal diagnosis of CF. ${ }^{3}$

Recommendations for population-based carrier screening for CF were published in 2001 by the American College of Medical Genetics (ACMG) in combination with the American College of Obstetricians and Gynecologists (ACOG) using a panel of 25 mutations. ${ }^{8}$ Initial technical guidelines for population-based CF screening were published in $2002 .{ }^{9}$ The initial 25 -mutation panel was modified in 2004, removing two mutations when it became clear that I148T was a polymorphism, and 1078delT was present in $<0.1 \%$ of $\mathrm{CF}$ chromosomes. ${ }^{10}$

The ACMG/ACOG recommendations selected mutations known to cause classical $\mathrm{CF}$ and excluded mutations causing mild CF. The R117H (c.350G > A) mutation, which by itself causes mild $\mathrm{CF}$ but when syntenic with a $5 \mathrm{~T}$ intron 8 -variant allele becomes a severe CF mutation, was given special consideration. In the original recommendations and in the revision, the screening committee stressed that only when $\mathrm{R} 117 \mathrm{H}$ is present with the $5 \mathrm{~T}$ variant should the individual be identified as a CF carrier. ${ }^{9,10}$

The current panel of 23 mutations represents the standard of care for CF screening. It is endorsed by ACMG, ACOG, National Institutes of Health, and the National Human Genome Research Institute. The testing landscape has become confounded by the advent of Food and Drug Administration-approved test kits that examine more than the 23 recommended mutations, and the availability of "expanded" mutation panels offered by several commercial laboratories consisting of several multiples of the ACMG/ ACOG core panel.

Our laboratory began offering $\mathrm{CF}$ testing using the core ACMG/ACOG panel of 23 mutations in 2002 and extensive gene sequencing since 2004. From 2002 to 2009, we reported the core panel mutations plus an additional eight mutations assayed by the testing reagent we were using. Beginning in June 2009, another mutation was added when we began using an Food and Drug Administration-approved test kit that includes 
the core panel plus an additional nine mutations. We reviewed our database of approximately 3 million panel tests and 2400 sequencing analyses to evaluate the performance of the ACMG/ ACOG panel and compare it to the performance of expanded panel screening.

\section{METHODS}

\section{ACMG/ACOG panel testing}

All panel testing was performed at either of two Quest Diagnostics Laboratories: Nichols Institute, San Juan Capistrano, CA, or Nichols Institute, Chantilly, VA. From July 2001 to December 2002, we used a LiPa strip analyte-specific reagent assay from Roche Molecular Systems that assayed the original 25 core ACMG/ACOG panel mutations. ${ }^{11}$ From December 2002 to June 2009, we used an analyte-specific reagent from Celera/Abbott that assayed 31 mutations. ${ }^{11,12}$ After June 2009, we used the Celera/Abbott In Vitro Diagnostic kit for assaying 32 mutations comprising the core 23 mutations in the revised ACMG/ACOG panel and nine additional mutations.

\section{Extensive sequencing}

Extensive sequencing of all exons and intron/exon junctions was performed as described previously. ${ }^{13}$ We received a clinical indication for 2393 patients samples submitted for extensive CFTR sequencing. The indications were newborn screening elevated IRT (209), diagnosis of CF (246), atypical (mild) CF (1557), carrier screening (251), and family history (130). We highly recommend that all samples be tested on a mutation panel before sequencing to avoid unnecessary sequencing costs. However, sometimes the ordering physician insists on moving directly to sequencing. When mutations were identified, we classified them into the following categories: mutations on the ACMG/ACOG core panel (ACMG), a mutation on a commonly ordered 97-mutation expanded panel (expanded), a mutation that is neither on the ACMG/ACOG panel nor on the 96mutation expanded panel (non-ACMG expanded), and D1152H (c.3454G > C), a mild CF mutation or polymorphism that is on most expanded panels but has extremely variable penetrance (see "Discussion").

\section{Data analysis}

All genotype information from the panel screening assay is stored contemporaneously in a proprietary database that is also used for reporting. All individuals with two panel mutations were removed because these patients were almost certainly referred for diagnostic testing and not carrier screening; 2,975,649 genotypes remained available for analysis. The assumption that all panel tests were submitted for populationbased carrier screening may lead to a small bias because some samples may have been from relatives of patients or known carriers or symptomatic individuals. We do not capture the indication for testing in our database. Therefore, we have no way to determine the percentage of panel testing samples were submitted for these indications, but we assume the overwhelming majority were from patients participating in populationbased carrier screening programs.

\section{RESULTS}

\section{General performance}

The overall carrier frequency observed in our 2,975,649 US pan-ethnic tests (Table 1) was 1:37.6. This is a $77 \%$ detection rate given the estimated US pan-ethnic carrier frequency of
Table 1 Frequencies of CF mutations detected in $2,975,649$ tests

\begin{tabular}{|c|c|c|c|}
\hline $\begin{array}{l}\text { Allele Common } \\
\text { (Standardized) }\end{array}$ & $\begin{array}{c}\text { Number } \\
\text { Chromosomes }\end{array}$ & $\begin{array}{l}\text { Percent of CF } \\
\text { chromosomes }\end{array}$ & Frequency \\
\hline Wild type & 5863532 & NA & \\
\hline $\begin{array}{l}\text { Delta F508 } \\
\text { (c.1520-1522delTCT) }\end{array}$ & 59194 & $75 \%$ & $1: 101$ \\
\hline $\begin{array}{l}\mathrm{R} 117 \mathrm{H}(\mathrm{c} .350 \mathrm{G}>\mathrm{A}) \\
\text { without } 5 \mathrm{~T}\end{array}$ & 9677 & NA & $1: 615$ \\
\hline G542X (c.1624G > T) & 2718 & $3.4 \%$ & $1: 2190$ \\
\hline $\mathrm{W} 1282 \mathrm{X}(\mathrm{c} .3846 \mathrm{G}>\mathrm{A})$ & 2464 & $3.1 \%$ & $1: 2415$ \\
\hline G551D (c.1652G > A) & 1891 & $2.4 \%$ & $1: 3147$ \\
\hline N1303K (c.3909C > G) & 1437 & $1.8 \%$ & $1: 4141$ \\
\hline $\begin{array}{l}3849+10 \mathrm{kbC}>\mathrm{T}(3717 \\
\quad+12191 \mathrm{C}>\mathrm{T})\end{array}$ & 1225 & $1.6 \%$ & $1: 4858$ \\
\hline $\mathrm{R} 117 \mathrm{H}+5 \mathrm{~T} / 2$ & 991 & $1.3 \%$ & $1: 6032$ \\
\hline $\begin{array}{l}3120+1 \mathrm{G}>\mathrm{A}(\mathrm{c} .2988 \\
\quad+198 \mathrm{G}>\mathrm{A})\end{array}$ & 974 & $1.2 \%$ & $1: 6110$ \\
\hline R553X (c.1657C > T) & 883 & $1.1 \%$ & $1: 6740$ \\
\hline $\begin{array}{l}1717-1 \mathrm{G}>\mathrm{A}(\mathrm{c} .1585 \\
-1 \mathrm{G}>\mathrm{A})\end{array}$ & 738 & $0.93 \%$ & $1: 8064$ \\
\hline $\begin{array}{l}621+1 \mathrm{G}>\mathrm{T}(\mathrm{c} .489 \\
\quad+1 \mathrm{G}>\mathrm{T})\end{array}$ & 735 & $0.93 \%$ & $1: 8097$ \\
\hline $\begin{array}{l}2789+5 \mathrm{G}>\mathrm{A}(\mathrm{c} .2657 \\
\quad+5 \mathrm{G}>\mathrm{A})\end{array}$ & 734 & $0.93 \%$ & $1: 8108$ \\
\hline $\begin{array}{l}\text { Delta I507 } \\
\text { (c.1519-1521delATC) }\end{array}$ & 508 & $0.64 \%$ & $1: 11715$ \\
\hline R334W (c.1000C > T) & 488 & $0.62 \%$ & $1: 12195$ \\
\hline G85E (c.254G > A) & 447 & $0.57 \%$ & $1: 13314$ \\
\hline $\mathrm{R} 1162 \mathrm{X}(\mathrm{c} .3484 \mathrm{C}>\mathrm{T})$ & 430 & $0.54 \%$ & $1: 13840$ \\
\hline $\mathrm{R} 347 \mathrm{H}^{a}(\mathrm{c} .1040 \mathrm{G}>\mathrm{A})$ & 332 & $0.42 \%$ & $1: 17926$ \\
\hline 3659delC (c.3528delC) & 328 & $0.41 \%$ & $1: 18144$ \\
\hline R560T (c.1679G > C) & 291 & $0.37 \%$ & $1: 20451$ \\
\hline $\begin{array}{l}1898+1 \mathrm{G}>\mathrm{A}(\mathrm{c} .1766 \\
\quad+152 \mathrm{G}>\mathrm{A})\end{array}$ & 287 & $0.36 \%$ & $1: 20736$ \\
\hline R347P (c.1040G > C) & 260 & $0.33 \%$ & $1: 22890$ \\
\hline $\mathrm{A} 455 \mathrm{E}(\mathrm{c} .1364 \mathrm{C}>\mathrm{A})$ & 256 & $0.32 \%$ & $1: 23247$ \\
\hline $3876 \operatorname{del}^{a}$ (c.3744delA) & 237 & $0.30 \%$ & $1: 25111$ \\
\hline $\mathrm{S} 549 \mathrm{~N}^{a}(\mathrm{c} .1646 \mathrm{G}>\mathrm{A})$ & 223 & $0.28 \%$ & $1: 26687$ \\
\hline $\begin{array}{l}711+1 \mathrm{G}>\mathrm{T}(\mathrm{c} .579 \\
+1 \mathrm{G}>\mathrm{A})\end{array}$ & 200 & $0.25 \%$ & $1: 29756$ \\
\hline 3905insT ${ }^{a}$ (c.3773-3774insT) & 160 & $0.20 \%$ & 1:37196 \\
\hline 394delTT ${ }^{a}$ (c.262-263delTT) & 151 & $0.19 \%$ & $1: 39413$ \\
\hline 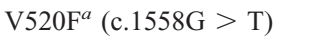 & 140 & $0.18 \%$ & 1:42509 \\
\hline 2184delA (c.2052delA) & 139 & $0.18 \%$ & $1: 42815$ \\
\hline 1078delT ${ }^{b}$ (c.949delT) & 116 & $0.15 \%$ & 1:51304 \\
\hline $\mathrm{S}_{549 \mathrm{R}^{a}}(\mathrm{c} .1645 \mathrm{~A}>\mathrm{C})$ & 77 & $0.097 \%$ & $1: 77290$ \\
\hline $\begin{array}{l}2183 \mathrm{AA}>\mathrm{G}^{a} \\
\quad(\text { c. } 2051 \mathrm{AA}>\mathrm{G})\end{array}$ & 17 & $\mathrm{NA}^{c}$ & $\mathrm{NA}^{c}$ \\
\hline
\end{tabular}

${ }^{a}$ Not on the ACMG recommended panel. ${ }^{10}$

${ }^{b}$ On original ACMG panel and removed in 2004 revision. ${ }^{10}$

${ }^{c}$ Only tested for 6 months so frequency cannot be determined from these data. 
$1: 29 .^{2}$ Of the 79,071 mutant alleles detected, 77,479 (98\%) were mutations in the core ACMG/ACOG panel. Limiting calculation to the mutations in the ACMG core panel yields a carrier frequency rate of 1:38.4, representing a $76 \%$ detection rate.

Our screening program data predict that approximately 1 in 1149 couples would be identified as at risk for conceiving a child with CF. If all these at-risk couples chose prenatal diagnosis, and the samples were referred to our laboratory, we would have expected 504 affected fetuses to be diagnosed.

We have performed 1310 prenatal diagnoses for CF since the inception of our program. The genotype results are 832 homozygous wild type, 359 heterozygotes, and 199 affected. The non-Hardy Weinberg distribution for the data is explained by the many prenatal tests for $\mathrm{CF}$ referred for reasons other than two carrier parents; for example, ultrasound findings of echogenic bowel. In our experience, these studies are almost always negative. A study of 682 cases of prenatal diagnosis for echogenic bowel revealed only $3 \%$ of fetuses with CF. ${ }^{14}$ Using the number of 199 affected fetuses identified as a starting point and the fact that $25 \%$ of fetuses will be affected when both parents are carriers, we estimate that 796 carrier couples elected to have prenatal diagnosis. We have no way of knowing how many of these couples were identified by the screening program versus couples who have had a previously affected child. Given our prediction that $1: 1149$ couples would be identified by panel screening (see earlier), this leads to a calculation of $796 / 1149$ or $53 \%$ of carrier couples who opted for prenatal diagnosis. Although there may be some ascertainment bias in terms of referral patterns for carrier versus prenatal diagnosis, these data indicate that two thirds of at-risk couples choose to proceed with prenatal diagnosis. Our data yield a ratio of one affected fetus for approximately 25,000 carrier screens.

\section{Ethnic-specific mutation data}

We request that the ordering physician or patient specify ethnicity when CF testing is ordered. If submitted, this information is stored in our database. In all, we received ethnicity data for $1,192,353$ or $60 \%$ of cases. Table 2 is a summary of the carrier frequencies in these various ethnic groups. The percentages of various ethnic groups in our pan-ethnic population were whites 64\%, Hispanics 16\%, African Americans 14\%, Asians 5\%, and Ashkenazi Jews $1 \%$. The final column shows the observed performance of the core panel testing compared with the estimates in the 2001 joint ACMG/ACOG guidelines ${ }^{8}$ and the revised ACOG committee report published in December 2005. ${ }^{15}$
The initial recommendations for population-based CF carrier screening targeted the two highest risk ethnic populations in the United States: whites and Ashkenazi Jews. For the 757,198 individuals self-reported as white, our 32-mutation panel identified 1:29 or approximately $86 \%$. The 2001 consensus statement predicted an $80 \%$ detection rate 8 ; ACOG 2005 revision anticipated an $88 \%$ detection rate. ${ }^{15}$

In the second targeted population, Ashkenazi Jews, the detection rate for 11,496 individuals was 1:27 or an $88 \%$ detection rate, lower than predicted but certainly adequate for screening purposes. The combined performance for these two ethnic groups is $>99 \%$ detection.

These data may be biased by the proportion of tests performed because of population-based carrier screening versus family history or symptoms (see "Methods"). Ashkenazi Jews, for example, have historically been users of population-based carrier screening for Tay-Sachs Disease and other disorders. However, the performance of close to $90 \%$ for both groups is gratifying given the initial targets were more modest for whites.

There has been much controversy regarding carrier detection sensitivity of the ACMG/ACOG panel for Hispanic Americans. In our series of 194,618 self-identified Hispanic Americans, we observed a CF carrier frequency of $1: 67$, leading to a $69 \%$ detection rate, far better than the initial prediction of $57 \%{ }^{8}$ and slightly lower than the ACOG estimate of $72 \% .{ }^{15}$

The observed performance of panel testing for the 163,389 African Americans in our series was 1:103, a detection rate of $63 \%$ in this population; similar to the revised estimate.

As expected, the ACOG panel is less able to detect $\mathrm{CF}$ carriers in Asian Americans, with a detection rate of $43 \%$ in our series of 64,615 individuals. The carrier frequency for individuals identifying themselves as Sephardic Jews was 1:67. These data would indicate that Sephardic Jews could benefit from $\mathrm{CF}$ testing using the current panels.

\section{Allele frequencies}

Table 1 represents the allele frequencies in our series of carrier tests. All patients with two mutations were removed from this series (see "Methods"). For patients with R117H (c.1040G > A) only individuals who carried a 5T allele were counted as CF carriers and that number was divided by 2 because we have no method of determining whether the 5T was is cis or trans. As expected, delta-F508 (c.1520-1522delTCT) is implicated in the majority of CF chromosomes, with a frequency of 1:101 tests, and accounts for $75 \%$ of CF chromo-

Table 2 Performance of cystic fibrosis carrier screening by ethnicity

\begin{tabular}{|c|c|c|c|c|c|c|}
\hline & No. patients & $\begin{array}{l}\text { Carrier frequency } \\
\text { estimated }^{a}\end{array}$ & $\begin{array}{c}\text { Carrier frequency } \\
\text { observed }\end{array}$ & $\begin{array}{l}\% \text { detection } \\
\text { observed }\end{array}$ & $\begin{array}{c}\% \text { detection predicted } \\
2001^{b}\end{array}$ & $\begin{array}{c}\% \text { detection predicted } \\
2005^{a}\end{array}$ \\
\hline White & 757,198 & $1: 25$ & $1: 25$ & 86 & 80 & 88 \\
\hline Ashkenazi Jewish & 11,496 & $1: 24$ & $1: 27$ & 88 & 97 & 94 \\
\hline Hispanic & 194,618 & $1: 46$ & $1: 62$ & 75 & 69 & 72 \\
\hline African American & 163,389 & $1: 65$ & $1: 94$ & 63 & 76 & 65 \\
\hline Asian American & 64,615 & $1: 94$ & $1: 200$ & 43 & 30 & 49 \\
\hline Sephardic Jewish & 1037 & & $1: 67$ & & & \\
\hline \multicolumn{7}{|c|}{$\begin{array}{l}\text { Performance detection rates can be compared with rates predicted by the ACMG/ACOG Clinical and Laboratory Guidelines, October } 2001 \text { and the ACOG Committee } \\
\text { Opinion, December } 2005 \text {. } \\
\text { a ACOG Committee Opinion, December } 2005.15 \\
b_{\text {A }} \text { ACMG }\end{array}$} \\
\hline
\end{tabular}


Table 3 Mutations detected in 62 newborns with elevated IRT and elevated sweat chlorides

\begin{tabular}{|c|c|}
\hline \multicolumn{2}{|l|}{ Two mutations detected from 51 newborns $(82 \%)$} \\
\hline $\mathrm{ACMG}^{a} / \mathrm{ACMG}$ & $1(2 \%)$ \\
\hline ACMG/expanded panel ${ }^{b}$ & $1(2 \%)$ \\
\hline ACMG/non-ACMG expanded ${ }^{c}$ & $40(64 \%)$ \\
\hline Expanded panel/non-ACMG or expanded & $1(2 \%)$ \\
\hline Non-ACMG expanded/non-ACMG expanded & $7(11 \%)$ \\
\hline D1152H/ACMG & 0 \\
\hline D1152H/Non-ACMG expanded & $1(2 \%)$ \\
\hline \multicolumn{2}{|l|}{ One mutation detected from nine newborns $(15 \%)$} \\
\hline ACMG & $7(11 \%)$ \\
\hline Expanded panel & $1(2 \%)$ \\
\hline Non-ACMG/nonexpanded & $1(2 \%)$ \\
\hline \multicolumn{2}{|l|}{ No mutation detected from two newborns ( $3 \%)$} \\
\hline $\begin{array}{l}{ }^{a} \mathrm{ACMG} / \mathrm{ACOG} \text { panel mutations. } \\
{ }^{b} \text { Mutation on } 97-\text { mutation expanded mutation panel. } \\
{ }^{c} \text { Mutation on neither ACMG panel nor } 97 \text {-mutation exp }\end{array}$ & \\
\hline
\end{tabular}

somes. Only 16 mutations occur with a frequency of $>1: 15,000$ tests, and these are all represented in the core $23 \mathrm{ACMG} / \mathrm{ACOG}$ panel. The highest frequency nonpanel mutation is $\mathrm{R} 347 \mathrm{H}$ (c.1040G > A), a mutation at the same base as a panel mutation R347P (c.1040G > C). The lowest frequency mutation remaining on the panel is 2184delA, occurring with a frequency of 1:42,815 tests; it accounts for $0.18 \%$ of $\mathrm{CF}$ chromosomes.

\section{Extensive DNA sequencing}

\section{Newborn sequencing}

Newborn screening programs in most states perform one or two IRT measurements followed by sweat chloride analysis and mutation panel testing. This is usually sufficient to establish the diagnosis of $\mathrm{CF}$ and identify the causative mutations. When these modalities fail to unambiguously confirm the diagnosis and/or genotype, extensive sequence analysis of CFTR has become a valuable tool. This is because it is possible for $\mathrm{CF}$ carriers to have elevated IRT and equivocal sweat test results; sweat testing in the newborn period is difficult both technically (to obtain a sufficient amount of material) and interpretively, because newborn sweat chloride values can be transiently depressed. ${ }^{5-7}$ DNA sequencing is often necessary to establish a reasonable prognosis for anxious parents. Because almost all patients in this sequencing series have had prior panel testing, and panel testing detects approximately $85 \%$ of CF mutations in the pan-ethnic US population (see earlier), we estimate that approximately $70 \%$ of newborns with $\mathrm{CF}$ will have two $\mathrm{CF}$ mutations identified by the core panel screening. Therefore, we expect samples submitted for sequencing to comprise approximately $30 \%$ of actual cases. We have no way to determine whether any parents of these newborns had population-based carrier detection during their pregnancy. Data from our extensive sequencing analyses are presented below.

In our series, we had 62 newborns with proven CF, confirmed by elevated IRT and positive sweat tests. The mutations discovered are listed in Table 3 . The majority of newborns in
Table 4 Mutations detected in 35 newborns with elevated IRT and borderline sweat chlorides

Two mutations detected from 10 newborns (29\%)

$\mathrm{ACMG} / \mathrm{ACMG}^{a}$

0

ACMG/expanded panel ${ }^{b}$

0

ACMG/non-ACMG nonexpanded ${ }^{c}$

$9(31 \%)$

Expanded panel/non-ACMG or expanded

0

Non ACMG-expanded/non-ACMG expanded

One mutation detected from 20 newborns (57\%)

ACMG

$18(51 \%)$

Expanded

Non-ACMG/nonexpanded

No mutation detected $5(4 \%)$

Results are shown for each panel used for testing.

${ }^{a} \mathrm{ACMG} / \mathrm{ACOG}$ panel mutations.

${ }^{b}$ Mutation on 97-mutation expanded mutation panel.

${ }^{c}$ Mutation on neither ACMG panel nor 97-mutation expanded panel.

this category $(64 \%)$ were compound heterozygotes for an ACMG/ACOG panel mutation and a mutation not on any panel. The next most common genotype was compound heterozygosity for two mutations not on any panel (11\%). Eleven percent of individuals had only a single identifiable mutation, and two patients had no identifiable CFTR mutation. A single patient who had not had prior panel screening actually had two ACMG/ ACOG panel mutations. One patient was a compound heterozygote for a non-ACMG expanded mutation and D1152H (c.3454G > C). Only one was a compound heterozygote for an expanded panel mutation and an ACMG panel mutation and would have been identified if both parents had been tested using an expanded panel.

There were 35 newborns with elevated IRT who had borderline sweat chloride results (Table 4). In this group, only 10 patients $(29 \%)$ had two CFTR mutations, and none of them would have been detected by expanded panel screening of both parents.

Eighty newborns had no sweat test results reported to us (Table 5). Only 17 of these patients had two CFTR mutations identified, and two of them were compound heterozygotes for D1152H (c.3454G > C). One patient was a compound heterozygote for an expanded panel mutation and ACOG panel mutation.

Therefore, in the series of 177 samples submitted because of elevated IRT, 89 are presumed to be affected with CF because of either an elevated sweat chloride or two probable causative severe CFTR mutations. Of these, only two would have been detected if their parents had expanded panel screening. Because we estimate that our series represents approximately $30 \%$ of newborns with $\mathrm{CF}$, expanded panel screening for all parents would be predicted to detect an additional $0.7 \%$ of all newborns with CF. Even if extensive sequencing had been applied to all parents, three newborns would not have been detected (Tables 3-5).

\section{Sequencing of patients with CF}

We received 246 samples with the test indication of CF. All individuals in this category had reported elevated sweat chlo- 
Table 5 Mutations detected in 80 newborns with elevated IRT and no sweat chloride results

\begin{tabular}{|c|c|}
\hline \multicolumn{2}{|l|}{ Two mutations detected from 17 newborns (21\%) } \\
\hline $\mathrm{ACMG}^{a} /$ expanded panel ${ }^{b}$ & $1(1 \%)$ \\
\hline Non-ACMG expanded ${ }^{c}$ Non-ACMG expanded & $3(3 \%)$ \\
\hline ACMG/Non-ACMG expanded & $11(13 \%$ \\
\hline D1152H/Non-ACMG expanded & $1(1 \%)$ \\
\hline D1152H/ACMG & $1(1 \%)$ \\
\hline \multicolumn{2}{|l|}{ One mutation detected from 48 newborns $(60 \%)$} \\
\hline $\mathrm{ACMG}$ & $38(47 \%$ \\
\hline Non-ACMG expanded & $8(10 \%$ \\
\hline D1152H & $2(2 \%)$ \\
\hline \multicolumn{2}{|l|}{ No mutation detected from 15 newborns (19\%) } \\
\hline $\begin{array}{l}\text { Results are shown for each panel used for testing. } \\
{ }^{a} \mathrm{ACMG} / \mathrm{ACOG} \text { panel mutations. } \\
{ }^{b} \text { Mutation on } 97 \text {-mutation expanded mutation panel. } \\
{ }^{c} \text { Mutation on neither ACMG panel nor } 97 \text {-mutation exp }\end{array}$ & \\
\hline
\end{tabular}

rides, but we did not systematically obtain any further clinical data regarding the severity of the disease in these patients. The results of sequencing analysis for these patients is shown in Table 6. Of note is that $9 \%$ of patients had no detectable mutation by extensive sequencing, and $12 \%$ had only one CFTR mutation detected. It is possible that these patients have large rearrangements, but our experience with our deletion/duplication assay is that approximately $10 \%$ of $\mathrm{CF}$ chromosomes remain unidentified after comprehensive CFTR analysis that includes deletion/duplication analysis and extensive sequencing. ${ }^{16}$ It is likely that the individuals with no detected CFTR mutations have CF caused by another gene capable of producing a phenocopy of $\mathrm{CF}$, as demonstrated conclusively in $2002 .{ }^{17}$

Table 6 DNA sequencing results for 246 patients with the diagnosis of $\mathrm{CF}$

\begin{tabular}{lc}
\hline Two mutations detected from 195 patients $(79 \%)$ & $33(13 \%)$ \\
ACMG/ACMG $^{a}$ & $112(46 \%)$ \\
ACMG/non-ACMG expanded & \\
Non-ACMG expanded/non-ACMG expanded & $25(10 \%)$ \\
ACMG/expanded & \\
Expanded/non-ACMG expanded & $20(8 \%)$ \\
One mutation detected from 29 patients $(12 \%)$ & $5(2 \%)$ \\
ACMG & \\
Non-ACMG expanded & $21(8 \%)$ \\
Expanded & $7(10 \%)$ \\
\end{tabular}

No mutation detected from 22 patients (9\%)

Results are shown for each panel used for testing.

${ }^{a} \mathrm{ACMG} / \mathrm{ACOG}$ panel mutations.

${ }^{b}$ Mutation on neither ACMG panel nor 97-mutation expanded panel.

${ }^{c}$ Mutation on 97-mutation expanded mutation panel.
Table 7 DNA sequencing results for 1557 patients with the diagnosis of atypical CF

\begin{tabular}{lc}
\hline Two mutations detected from 148 patients $(10 \%)$ & \\
ACMG/ACMG $^{a}$ & $7(<1 \%)$ \\
ACMG/R117H + 7T & $2(<1 \%)$ \\
ACMG/Non-ACMG expanded ${ }^{b}$ & $75(4 \%)$ \\
Non-ACMG expanded/Non-ACMG expanded & $59(4 \%)$ \\
ACMG/expanded & \\
Expanded/non-ACMG expanded & $8(<1 \%)$
\end{tabular}

One mutation detected from 419 patients $(27 \%)$

ACMG

$160(10 \%)$

Non-ACMG expanded

Expanded

$3(<1 \%)$

No mutation detected from 990 patients (64\%)

Results are shown for each panel used for testing.

${ }^{a} \mathrm{ACMG} / \mathrm{ACOG}$ panel mutations.

${ }^{b}$ Mutation on neither ACMG panel nor 97-mutation expanded panel.

${ }^{c}$ Mutation on 97-mutation expanded mutation panel.

This finding reemphasizes that molecular methods cannot detect all CF cases.

The largest series of samples submitted for extensive sequencing is the 1557 samples with the diagnosis of atypical or mild CF (Table 7). Most patients (64\%) have no detectable CFTR mutations. Approximately $10 \%$ of patients have two demonstrable CFTR mutations, whereas 419 patients, or $27 \%$, have symptoms of atypical CF with a single demonstrable CFTR mutation. This raises the question whether carriers for $\mathrm{CF}$ may be mildly symptomatic under certain circumstances due to their CFTR genotype. Perhaps modifier genes or environmental factors can combine with a heterozygous CFTR genotype to cause atypical CF symptoms (see "Discussion").

\section{DISCUSSION}

Our data found that $27 \%$ of individuals with the diagnosis of atypical or mild CF have a single CFTR mutation. This suggests that some heterozygous carriers are mildly symptomatic. The expected carrier frequency in the pan-ethnic US population is $1: 29$ or $3 \%$. Therefore, the prevalence of $27 \%$ represents an approximate 8-fold increase above the expected frequency in this series of patients with symptoms associated with mild CF. Previous reports have demonstrated statistically significant higher $\mathrm{CF}$ carrier rates in children with chronic rhinosinusitis, and rhinosinusitis than in the general population. ${ }^{18,19} \mathrm{~A}$ higher incidence of rhinosinusitis was demonstrated in an analysis of individuals who were obligate $\mathrm{CF}$ carriers. ${ }^{20}$ Chronic pancreatitis has also been associated with CF carriers in two large studies. ${ }^{21,22}$ At least one study has failed to replicate an increased incidence of CF-related symptoms in obligate carriers. ${ }^{23}$ Our data strongly support the hypothesis that some CF carriers are symptomatic due to their genotype. Further evidence that heterozygous CF carriers can have phenotypic effects is the data from newborn screening programs that demonstrate that carriers can have elevated IRT in the newborn period from blockage of their pancreatic ducts. 5,6 
CFTR heterozygotes being mildly symptomatic is one possible explanation for the fact that $27 \%$ of patients with mild CF have only one mutation, but there are others: the role of mutations or polymorphisms in regulatory regions, modifier genes, or just an incorrect diagnosis (i.e., a different cause for their respiratory or digestive symptoms). Modifying genes are as likely to act on individuals with one mutation as they are in individuals with two mutations, as discussed earlier in this study. Furthermore, there are many more carriers identified through carrier screening with no discernible symptoms. The cases discussed in this study were ascertained through their symptoms. This can lead to ascertainment bias: they are more likely to have a mutation if they have symptoms than if they do not but that does not make the inverse true: we cannot extrapolate that those who have a mutation (are heterozygotes) are more likely to have symptoms. The best way to determine the frequency of symptoms in carriers is to assess individuals identified as carriers through population-based screening and subsequently assess them for CF-related symptoms.

There were seven patients in our sequencing series with the diagnosis of atypical CF who actually had two ACMG/ACOG panel mutations. This may indicate that there is some variable phenotypic expression even when two severe CF mutations are present. Several modifying loci have been described that may help explain this phenomenon. ${ }^{24}$

These data demonstrate that no current molecular method is capable of detecting all newborns with $\mathrm{CF}$ and that the sweat test must remain an essential element in the diagnosis of $\mathrm{CF}$, as emphasized by Farrell et al. ${ }^{3}$ It must also be noted that we cannot determine the severity of CF by newborn screening results; some of these newborns may have mild or atypical disease.

We examined the performance of the ACMG/ACOG core mutation panel in population-based carrier screening in the United States using a database of approximately 3 million carrier screens and more than 2000 extensive sequencing analyses. The data demonstrate that the core ACMG/ACOG mutation panel is performing as predicted, identifying approximately $90 \%$ non-Hispanic white CF carriers and $77 \%$ of CF carriers in a US pan-ethnic population consisting of a mixture of $64 \%$ non-Hispanic whites, 16\% Hispanic Americans 14\% African Americans, 5\% Asian Americans, and 1\% Ashkenazi Jews. We must acknowledge a potential bias leading to an overestimation as discussed in the Methods section, but our data do not allow us to investigate the potential contribution of this bias.

A particularly contentious aspect of the debate regarding the number of mutations to include on a US screening panel is detection rates in Hispanic Americans. ${ }^{25}$ This is fueled by two factors: the initially low estimate of $57 \%$ detection in the joint ACMG/ACOG practice guideline 8 and the inclusion of mutations on expanded panels that are either polymorphisms or mild mutations with a high prevalence in Hispanic Americans. Our data demonstrate that the ACMG/ACOG core panel actually detects $75 \%$ of mutations in the Hispanic American population.

In a publication in 2004, Sugarman et al. ${ }^{26}$ reported on their improved performance for detection of CF carriers in the Hispanic American population using a panel of 87 mutations. The increased detection rates were primarily due to mutations D1152H (c.3454G > C) detected in 18 patients accounting for $6.27 \%$ of Hispanic CF chromosomes and L206W (c.617T > G) detected in 11 patients and comprising 3.83\% of Hispanic CF chromosomes. Several studies have demonstrated that neither of these mutations are severe CF mutations.

In a study of 91 patients with $\mathrm{D} 1152 \mathrm{H}$ and the diagnosis of CF, Massaffi et al. ${ }^{27}$ state, "Although comprising $5-6 \%$ of mutations on genetic screening, clinical reports of cystic fibrosis (CF) are rare, suggesting that the disease is mild, atypical, or even absent." A second publication demonstrated that for 42 subjects who were compound heterozygotes for D1152H and a classic CF mutation, the median age at diagnosis was 33 years, and 11 patients had CBAVD as their only presenting complaint. ${ }^{28}$ The $\mathrm{CF}$ mutation database describes a single proband as follows: the family in whom this mutation was identified is of Ashkenazi/Jewish/North European descent and is remarkable for advanced age, mild pulmonary disease, pancreatic sufficiency, and normal sweat chloride values. The index patient was diagnosed with variant $\mathrm{CF}$ at the age of 57 years on the basis of clinical phenotype and abnormal nasal epithelial bioelectric parameters. ${ }^{29}$

The variant D1152H is likely similar to I148T. Because of its high prevalence in certain populations, it is present in some patients with $\mathrm{CF}$ because of a rare linkage with a true $\mathrm{CF}$ causing mutation. Alternatively, D1152H may be analogous to $\mathrm{R} 117 \mathrm{H}$, causing mild CF by itself but requiring a second mutation before becoming a severe CF mutation. Until the disease association is better understood, D1152H should not be part of a population-based $\mathrm{CF}$ carrier screening program.

The second mutation, L206W (c.617T > G), is similar to $\mathrm{D} 1152 \mathrm{H}$. This is another variant with high prevalence in certain populations and documented atypical presentations. After sequencing 40 CBAVD patients, Bassas et al. ${ }^{30}$ concluded that L206W, R74W/D1270N, and R117H are benign $\mathrm{CF}$ mutations. Another report described four patients with L206W and mild CF. 31

In the study by Sugarman et al., ${ }^{26}$ neither D1152H nor L206W were found in 159 Hispanic patients with CF providing further evidence of the mild nature of these alleles. These two mutations were only detected in the carrier-screened population at high frequencies. If these two mutations were CF-causing mutations and were found in a combined $10 \%$ of Hispanic CF chromosomes, it is inconceivable that it would not be detected even a single time in 159 Hispanic patients with CF.

It is unmistakable from the initial guidelines that populationbased carrier screening was specifically designed to exclude the detection of mild or variable alleles. The requirement that $\mathrm{R} 117 \mathrm{H}$ (c.350G > A) be reported only when syntenic with a $5 \mathrm{~T}$ was intended to avoid creating a dilemma for parents, which could result in the termination of a fetus with CBAVD alone or other mild forms of CF. It is impossible to confirm the diagnosis of $\mathrm{CF}$ in utero nor to predict or assess the potential severity of the disease. Analyzing and reporting D1152H and L206W as $\mathrm{CF}$ mutations in a population-based carrier screening program and reporting heterozygous individuals to be $\mathrm{CF}$ carriers raises the specter of parents terminating unaffected or mildly affected fetuses due to fears of having a child affected with classical CF. By using the prevalence of these variants to claim increased detection rates in Hispanic Americans, some commercial laboratories are violating the precepts of population-based CF carrier screening.

An examination of allele frequencies in Table 1 demonstrates that 21 of the 22 most prevalent CF mutations are on the ACMG/ACOG core panel. The only mutation not on the core panel that occurs with a frequency of $>1: 25,000$ tests is $\mathrm{R} 347 \mathrm{H}$ (c.1040G > A). Since R347H occurs at the same base as the panel mutation R347P (c.1040G > C), most testing systems are already detecting this mutation. Even if a testing system does not detect $\mathrm{R} 347 \mathrm{H}$, this mutation only accounts for $0.38 \%$ of $\mathrm{CF}$ chromosomes.

In more than 250 sequencing analyses of newborns with proven $\mathrm{CF}$ and known patients with $\mathrm{CF}$, we have not seen a 
single rare variant more than three times. This indicates that there are no frequent founder mutations in the US population not represented on the ACMG/ACOG core panel that should be added to the current panel. Although a recurrent 21-kb Slavic deletion allele was described (del2,3 [21kb]), ${ }^{31}$ this mutation was observed in only $0.2 \%$ of the US population. ${ }^{32}$ Given the uncertainties with respect to identification of ethnicity and the logistical problems of having different ethnic-specific panels, we feel that creating a battery of ethnic-specific panels is undesirable. In our sequencing experience, no mutation has been found more than three times in the pan-ethnic US population. The frequency of the the $21 \mathrm{~kb}$ deletion in the pan-ethnic US population is insufficient for inclusion into the current US recommended screening panel. Therefore, altering the core panel to include this mutation seems unnecessary at this time.

In conclusion, our review found that the revised ACMG/ ACOG core mutation panel is performing far better than expected in detecting carriers of classical $\mathrm{CF}$ and there is no compelling reason to consider changing current recommendations at this time.

\section{REFERENCES}

1. Cystic Fibrosis Foundation Web Page. Available at: http://www.cff.org/ AboutCF/. Accessed May 21, 2010.

2. Cystic Fibrosis Foundation Web Page. Available at: http://www.cff.org/ AboutCF/Testing/GeneticCarrierTest/. Accessed May 21, 2010.

3. Farrell PM, Rosenstein BJ, White TB, et al. Guidelines for diagnosis of cystic fibrosis in newborns through older adults: Cystic Fibrosis Foundation consensus report. J Pediatr 2008;153:S4-S14.

4. Rowe, SM, Miller S, Sorscher EJ. Cystic fibrosis. N Engl J Med 2005;352: 1992-2001.

5. Parad RB, Comeau AM. Diagnostic dilemmas resulting from the immunoreactive trypsinogen/DNA cystic fibrosis newborn screening algorithm. $J$ Pediatr 2005;147:S78-S82.

6. Castellani C, Picci L, Scarpa M, et al. Cystic fibrosis carriers have higher neonatal immunoreactive trypsinogen values than non-carriers. Am J Med Genet A 2005; 135:142-144.

7. Parad RB, Comeau AM, Dorkin HL, et al. Sweat testing infants detected by cystic fibrosis newborn screening. J Pediatr 2005;147:S69-S72.

8. American College of Obstetricians and Gynecologists and American College of Medical Genetics. Preconception and prenatal carrier screening for cystic fibrosis, clinical and laboratory guidelines. Washington DC: American College of Obstetricians and Gynecologists, 2001.

9. Richards CS, Bradley LA, Amos J, et al. Standards and guidelines for CFTR mutation testing. Genet Med 2002;4:379-391.

10. Watson MS, Cutting GR, Desnick RJ, et al. Cystic fibrosis population carrier screening: 2004 revision of the American College of Medical Genetics mutation panel. Genet Med 2004;6:387-391.

11. Strom CM, Huang D, Buller A, et al. Cystic fibrosis screening using the college panel: platform comparison and lessons learned from the first 20,000 samples. Genetic Med 2002;4:289-296.
12. Strom CM, Crossley B, Redman JB, et al. Cystic fibrosis screening: lessons learned from the first 320,000 patients. Genet Med 2004;6:136-140.

13. Strom CM, Huang D, Chen C, et al. Extensive sequencing of the cystic fibrosis transmembrane regulator gene: assay validation and unexpected benefits of developing a comprehensive test. Genet Med 2003;5:9-14.

14. Simon-Bouy B, Satre V, Ferec C, et al. Hyperechogenic fetal bowel: a large French collaborative study of 682 cases. Am J Med Genet A 2003;121A: 209-213.

15. Committee on Genetics, American College of Obstetricians and Gynecologists. ACOG Committee Opinion. Number 325, December, 2005. Update on carrier screening for cystic fibrosis. Obstet Gynecol 2005;106:1465-1468.

16. Hantash FM, Redman JB, Starn K, et al. Novel and recurrent rearrangements in the CFTR gene: clinical and laboratory implications for cystic fibrosis screening. Hum Genet 2006;119:126-136.

17. Groman JD, Meyer ME, Wilmott RW, Zeitlin PL, Cutting GR. Variant cystic fibrosis phenotypes in the absence of CFTR mutations. $N$ Engl J Med 2002;347:401-407.

18. Raman V, Clary R, Siegrist KL, Zehnbauer B, Chatila TA. Increased prevalence of mutations in the cystic fibrosis transmembrane conductance regulator in children with chronic rhinosinusitis. Pediatrics 2002; 109:E13.

19. Wang X, Moylan B, Leopold DA, et al. Mutation in the gene responsible for cystic fibrosis and predisposition to chronic rhinosinusitis in the general population. JAMA 2000;284:1814-1819.

20. Wang X, Kim J, McWilliams R, Cutting GR. Increased prevalence of chronic rhinosinusitis in carriers of a cystic fibrosis mutation. Arch Otolaryngol Head Neck Surg 2005;131:237-240.

21. Sharer N, Schwartz M, Malone G, et al. Mutations of the cystic fibrosis gene in patients with chronic pancreatitis. N Engl J Med 1998;339:645-652.

22. de Cid R, Ramos MD, Aparisi L, et al. Independent contribution of common CFTR variants to chronic pancreatitis. Pancreas 2010;39:209-215.

23. Castellani C, Quinzii C, Altieri S, Mastella G, Assael BM. A pilot survey of cystic fibrosis clinical manifestations in CFTR mutation heterozygotes. Genet Test 2001;5:249-254.

24. Collaco JM, Cutting GR. Update on gene modifiers in cystic fibrosis. Curr Opin Pulm Med 2008;14:559-566.

25. Grody WW, Cutting GR, Watson MS. The cystic fibrosis mutation "arms race": when less is more. Genet Med 2007;9:739-744.

26. Sugarman EA, Rohlfs EM, Silverman LM, Allitto BA. CFTR mutation distribution among US Hispanic and African American individuals: evaluation in cystic fibrosis patient and carrier screening populations. Genet Med 2004;6:392-399.

27. Massaffi H, Prais D, Mei-Zahav M, Blau H. Cystic fibrosis mutations with widely variable phenotype: the D1152H example. Pediatr Pulmonol 2006; 41:250-254.

28. Burgel PR, Fajac I, Hubert D, et al. Non-classic cystic fibrosis associated with the D1152H CFTR mutation. Clin Genet 2010;77:355-364.

29. Toronto Sick Childrens CF mutation database. Available at: http://www. genet.sickkids.on.ca. Accessed May 24, 2001.

30. Casals T, Bassas L, Ruiz-Romero J, et al. Extensive analysis of 40 infertile patients with congenital absence of the vas deferens: in $50 \%$ of cases only one CFTR allele could be detected. Hum Genet 1995;95:205-211.

31. Desgeorges M, Rodier M, Piot M, Demaille J, Claustres M. Four adult patients with the missense mutation L206W and a mild cystic fibrosis phenotype. Hum Genet 1995;96:717-720.

32. Dörk T, Macek M Jr, Mekus F, et al. Characterization of a novel 21-kb deletion, CFTRdele2,3(21 kb), in the CFTR gene: a cystic fibrosis mutation of Slavic origin common in Central and East Europe. Hum Genet 2000;106:259-268.

\section{Note from the Authors}

Our article, Cystic fibrosis testing 8 years on: Lessons learned from carrier screening and sequencing analysis, was published online ahead of print and available from November to December 2010. It was called to our attention that in Table 1, the incidence of $\mathrm{R} 117 \mathrm{H}+5 \mathrm{~T}$ was approximately 10 fold higher than in all previous reports. This triggered a re-analysis of our data that determined that the $\mathrm{R} 117 \mathrm{H}$ frequency reported in Table 1 was, in fact, all R117H regardless of the intron 8 status. This triggered a re-analysis of all data for Tables 1 and 2 and these results altered our original conclusion that the ACMG/ACOG panel was performing better than expected. In fact, the panel is performing as expected. No other data or conclusions of our article were altered. The current Electronic Publication and the article in the print version of Genetics in Medicine contain the corrected figures and conclusions. We deeply regret this error. 